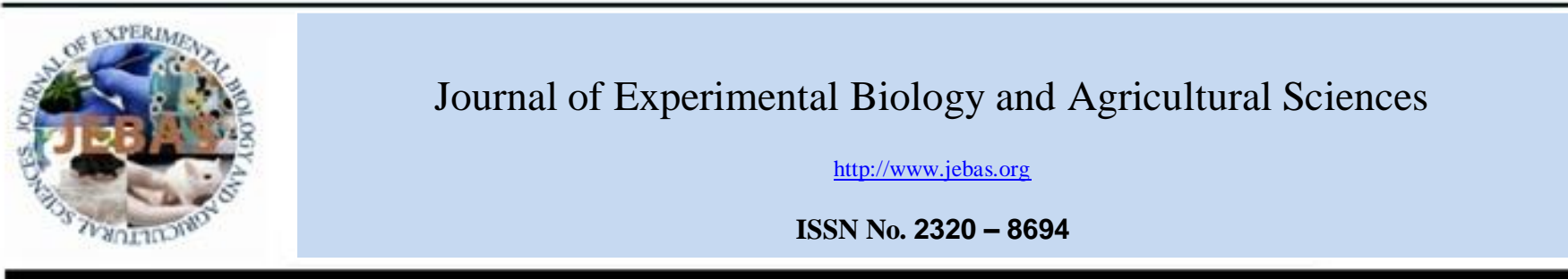

\title{
LOCALIZATION OF PHOSPHATASES, OXIDOREDUCTASES AND ESTERASES IN DIFFERENT SEGMENTS OF OVIDUCT IN LAYING PUNJAB WHITE QUAIL
}

\section{Sukhadeve S.V.* and Neelam Bansal}

Department of Veterinary Anatomy, GADVASU, Ludhiana (Punjab) - 141004

Received - April 07, 2017; Revision - July 28, 2017; Accepted - August 27, 2017

Available Online - September 10, 2017

DOI: http://dx.doi.org/10.18006/2017.5(4).556.560

KEYWORDS

Enzymes

Oviduct

Punjab white quail

\section{ABSTRACT}

The present study was conducted to observe the distribution pattern of phosphatases, oxidoreductases and esterases in different segments of oviduct in Punjab white quail. The study depicted that the activity of alkaline phosphatase was moderate to strong in propria submucosa of all the segments of the oviduct, while negligible to weak in other layers. The SDH, LDH and G-6-PD activity was weak to moderate in lamina epithelialis in all segments except magnum where it was negligible to weak. NADH had stronger reaction in lamina epithelialis of infundibulum,whereas NADPH showed weak to moderate activity in lamina epithelialis and moderate in propria submucosa of infundibulum and magnum. The reaction of non specific esterase in lamina epithelialis was weak to moderate in the infundibulum, magnum, uterus and vagina whereas moderate to strong activity in isthmus while propria submucosa showed moderate activity in infundibulum, uterus and vagina and weak in magnum and isthmus. The infundibular, magnal and sperm host glands showed weak to moderate activity of different enzymes studied. The variation in enzymatic activity may be correlated with the reproductive status of the Punjab white quail.
* Corresponding author

E-mail: drsuchits@gmail.com (Sukhadeve S.V.)

Peer review under responsibility of Journal of Experimental Biology and Agricultural Sciences.

Production and Hosting by Horizon Publisher India [HPI] (http://www.horizonpublisherindia.in/).

All rights reserved.
All the article published by Journal of Experimental Biology and Agricultural Sciences is licensed under a Creative Commons Attribution-NonCommercial 4.0 International License Based on a work at www.jebas.org.

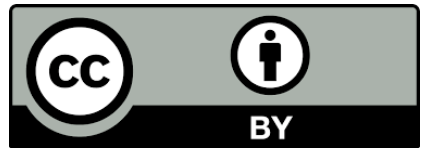




\section{Introduction}

The Punjab white quail is notably valuable egg and meat producer and also used as laboratory animal. The avian oviduct is complex biological organ that undergoes series of hormonal, neural, biochemical, cellular and enzymatic changes during formation of egg. The female reproductive tract is capable for fertilizing egg for days or weeks in many species (Baskt, 2011). Most of the studies have been conducted on histomorphological aspect in oviduct of Japanese quail, but scanty information available on localization of enzymes in oviduct of turkeys (Baskt \& Akuffo,2007), fowl (Aire \& Steinbach,1976), Japanese quail (Yamamoto et al., 1985; Khillare et al., 2013). So, the present work was planned to explore the localization in phosphatases, oxidoreductases and estarases in different segments of oviduct in Punjab white quail.

\section{Materials and Methods}

The fresh tissue samples from different segments of oviduct (infundibulum, magnum, isthmus, uterus, uterovaginal junction and vagina) were collected from 32 weeks old Punjab white quail immediately after sacrifice and kept at $-20^{\circ} \mathrm{C}$. Later, sections of 10 micron thickness were obtained by cryosectioning with cryostat microtme. The slides were incubated for the demonstration of phosphatases (alkaline phosphatase and glucose-6-phosphatase) by Barka \& Anderson (1963), dehydrogenases (lactate, succinate and glucose-6-phosphate dehydrogenase) and diaphorases (NADH and NADPH) as per standard protocol by Pearse (1972) and nonspecific esterase by Barka \& Anderson (1963). The observations were recorded by digital microscope with the image analysis system and necessary photomicrographs were taken.

\section{Results and Discussion}

The histoenzymic activity on different layers in the various segments of oviduct in Punjab white quail (PWQ) and with respect to phosphatases, oxidoreductases and estarases Table 1.

Table I Histoenzymatic activity in different segments of oviduct in Punjab white quail

\begin{tabular}{|c|c|c|c|c|c|c|c|c|}
\hline $\begin{array}{l}\text { Enzyme } \\
\text { Segment }\end{array}$ & AKPase & G6Pase & LDH & SDH & G6PD & NADH & NADPH & NSE \\
\hline \multicolumn{9}{|l|}{ Infundibulum } \\
\hline $\mathrm{LE}$ & $0 /+$ & $0 /+$ & $++/+++$ & $+/++$ & $+/++$ & $++/+++$ & ++ & $+/++$ \\
\hline PS & $++/+++$ & ++ & + & + & $+/++$ & ++ & ++ & ++ \\
\hline TM & $0 /+$ & 0 & $0 /+$ & $0 /+$ & + & + & + & + \\
\hline TS & $0 /+$ & 0 & $0 /+$ & $0 /+$ & + & + & + & + \\
\hline \multicolumn{9}{|l|}{ Magnum } \\
\hline LE & $0 /+$ & $+/++$ & $++/+++$ & $+/++$ & $+/++$ & $++/+++$ & ++ & ++ \\
\hline PS & $++/+++$ & ++ & $+/++$ & $0 /+$ & $0 /+$ & ++ & & + \\
\hline TM & 0 & $0 /+$ & $+/++$ & $0 /+$ & $0 /+$ & + & + & + \\
\hline TS & 0 & $0 /+$ & $+/++$ & $0 /+$ & $0 /+$ & + & + & + \\
\hline \multicolumn{9}{|l|}{ Isthmus } \\
\hline$\overline{L E}$ & $0 /+$ & $0 /+$ & $+/++$ & $++/+++$ & $0 /+$ & $++/+++$ & ++ & $++/+++$ \\
\hline PS & $++/+++$ & + & $+/++$ & $0 /+$ & $0 /+$ & + & & + \\
\hline TM & $0 /+$ & $0 /+$ & + & + & $0 /+$ & $0 /+$ & & ++ \\
\hline TS & $0 /+$ & $0 /+$ & + & + & $0 /+$ & $0 /+$ & & $++/+++$ \\
\hline \multicolumn{9}{|l|}{ Uterus } \\
\hline LE & $0 /+$ & $0 /+$ & $+/++$ & $++/+++$ & + & $+/++$ & & $+/++$ \\
\hline PS & $++/+++$ & + & + & + & + & ++ & ++ & \\
\hline $\mathrm{TM}$ & + & $0 /+$ & $0 /+$ & $0 /+$ & $0 /+$ & $+/++$ & & + \\
\hline $\mathrm{TS}$ & + & $0 /+$ & $0 /+$ & $0 /+$ & $0 /+$ & ++ & & + \\
\hline \multicolumn{9}{|l|}{ Vagina } \\
\hline LE & $+/++$ & $+/++$ & $+/++$ & $++/+++$ & + & $+/++$ & & $+/++$ \\
\hline PS & $++/+++$ & + & + & + & $0 /+$ & ++ & ++ & \\
\hline TM & + & + & $0 /+$ & $0 /+$ & 0 & + & $++/+++$ & + \\
\hline $\mathrm{TS}$ & + & + & $0 /+$ & $0 /+$ & 0 & + & $++/+++$ & + \\
\hline
\end{tabular}
LE-Lamina Epithelias
PS-Propria Submucosa TM- Tunica Muscularis
$0=$ Negative
0/+= Negligible to weak
$+=$ Weak $++=$ Modertate
TS- Tunica Serosa
$+++=$ Strong

Journal of Experimental Biology and Agriculture Science http://www.jebas.org 


\subsection{Alkaline Phosphatase (AKPase)}

The activity of AKPase was found to be moderate to strong in propria submucosa of all the segments of the oviduct, while negligible to weak in lamina epithelialis of infundibulum, magnum, isthmus and uterus but weak to moderate in vagina (Figure 2).Tunica muscularis and tunica serosa showed negligible to weak reaction in all the segments of oviduct. Strong to intense reaction was seen in the tunica intima of blood vessels present in the submucosa and lamina propria of infundibulum (Figure 1), magnum, isthmus and uterus, while vagina showed negligible to weak activity. This may be due to the more ionic movement in the endothelium of blood vessels. Presence of AKPase activity may be associated with cellular differentiation and proliferation during oviductal maturation as reported by Uppal \& Roy (2007) in buffalo fetus. The sperm host gland (SHG) present in the uterovaginal junction showed weak to moderate reaction. The variation in AKPase activity was also reported earlier in sperm storage tubules in fowl (Aire \& Steinbach, 1976), in oviducts of chicken (Bashir et al., 2002) and in Japanese quail (Khillare et al., 2013).

\subsection{Glucose-6-phosphatase (G-6-Pase)}

Negligible to weak activity observed in lamina epithelialis of all the segments of oviduct except in magnum and vagina where weak to moderate reaction was seen. The activity varied from weak to moderate in propria-submuosa of different segments while it was reported to be negligible to weak in tunica muscularis and tunica serosa.The activity was found to be more in the basal lamina of the glandular epithelium than the lumen of proprial gland in magnum and SHG in utero-vaginal junction (Figure 3).This indicates the low glucose metabolism in different segments. Similar activity of G-6-Pase was reported in oviduct of buffalo foetus by Uppal \& Roy (2007).

\subsection{Lactate dehydrogenase (LDH)}

Moderate to strong activity of LDH in lamina epithelialis of infundibulum and magnum while weak to moderate in isthmus, uterus (Figure 4) and vagina. Negligible to weak activity was seen in propria submucosa, tunica muscularis and tunica serosa of all segments except magnum where it was slightly stronger. Proprial glands located in caudal part of infundibulum and magnum showed weak activity but SHG had strong LDH reaction. Khillare (2012) also reported similar LDH activity in sperm storage tubules of Japanese quail. As LDH is an NAD dependent enzyme, so its presence in the cells may be due to active glycolytic pathway. It also catalyses the formation of lactate in anaerobic glycolysis and pyruvate in aerobic respiration.

\subsection{Succinate dehydrogenase (SDH)}

Lamina epithelialis showed weak to moderate SDH activity in infundibulum and magnum but moderate to strong in isthmus, uterus and vagina (Figure 5). The activity was varied negligible to weak in propria submucosa, tunica muscularis and tunica serosa of all the segments oviduct except in isthmus where it was weak to moderatein propris submucosa indicating low metabolism. The activity was more in the proprial glands of magnum than SHG. Similar SDH activity was demonstrated in shell glands in Japanese quail by Yamamoto et al. (1985), oviductular epithelium of rat (Padykula, 1952) and infranuclear part of epithelium in ampulla of the mouse (Fredricssion, 1969). According to Yamamoto et al. (1985), SDH estimates the role of mitochondria in calcium transport in avian shell glands.

\subsection{Glucose-6-phosphate dehydrogenase (G-6-PD)}

The activity of G-6-PD was weak to moderate in lamina epithelialis of infundibulum and magnum and propria submucosa of infundibulum while negligible to weak in propria submucosa, tunica muscularis and tunica serosa of other segments of oviduct (Figure 6). The activity was more in SHG glands than proprial glands of magnum and infundibulum. The presence of G-6-PD enzyme activity reflects the involvement of pentose phosphate shunt required for production and utilization of nucleic acids during cell growth. G-6-PD is involved in the pentose phosphate pathway and convert Glucose-6-phosphate into 6phosphogluconolactone which is necessary for the nucleic acid (ribose and deoxyribose) synthesis (Rama Rao, 1994).

\subsection{Reduced nicotinamide adenine dinucleotide diaphorase (NADH diaphorase)}

Lamina epithelialis showed a moderate to strong activity of $\mathrm{NADH}$ in infundibulum, magnum (Figure 7) and isthmus while weak to moderate reactivity in uterus and vagina. The reaction was more in the basal lamina of epithelial mucosa than apical border. Weak to moderate activity was observed in propria submucosa, tunica muscularis and tunica serosa of infundibulum, magnum, uterus and vagina, while tunica muscularis and tunica serosa of isthmus showed negligible to weak activity. The reaction was moderate in proprial glands of infundibulum, magnum and SHG. Strong activity may be indicative of higher metabolic activity in the cellular proliferation of lamina epithelialis. Similar finding have been reported in oviduct of buffalo foetus by Uppal \& Roy (2007).

\subsection{Reduced nicotinamide adenine dinucleotide phosphate diaphorase (NADPH diaphorase)}

NADPH activity was observed to be moderate to strong in lamina epithelialis of all segments of oviduct (Figure 8). Moderate activity was seen in propria submucosa of infundibulum, 
magnum, uterus and vagina but moderate to strong in isthmus. Tunica muscularis and tunica serosa showed weak activity in all segments except in vagina where it was moderate to strong. The glands of caudal infundibulum, magnum and utero-vaginal junction showed weak activity. NADPH is coenzyme of dehydrogenases and its activity reflects the metabolic status of the cell.

\subsection{Non specificestarase (NSE)}

The lamina epithelialis showed weak to moderate NSE activity in the infundibulum, magnum (Figure 9), uterus and vagina whereas moderate to strong in isthmus. Propria submucosa showed moderate activity in infundibulum, uterus and vagina while weak reaction in magnum and isthmus. Tunica muscularis and tunica serosa had weak reaction in all segments except isthmus which revealed moderate to strong activity. Moderate reaction was observed in glands of uterus and utero-vaginal junction while that of magnum showed negligible to weak reaction. Valocky et al. (2007) also reported NSE in the surface epithelial and glandular cells in oviduct of puerperal ewes. The activity of NSE may be due to its involvement in the process of energy transformation.

It may be concluded from the present study that there is variation in the activity of phosphatises, oxidoreducatses and esterases in different segments of oviduct in Punjab white quail. This may be due to the variation in cellular differentiation and proliferation of oviduct and reproductive status in Punjab white quails.
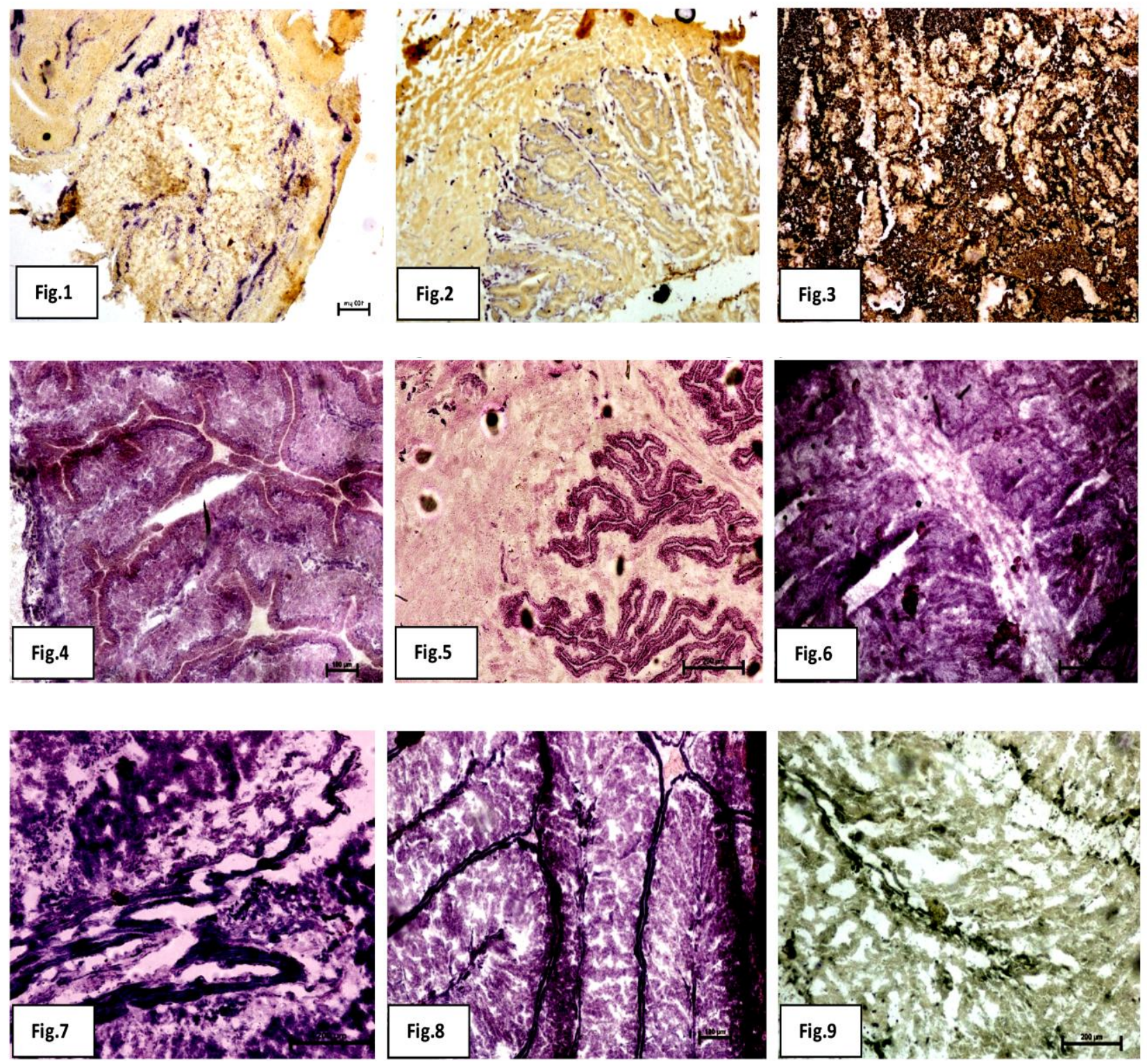

Journal of Experimental Biology and Agriculture Science http://www.jebas.org 
Legends for the photographs: Cryosections of oviduct of different segments showing:

Figure 1 Strong to intense positive reaction AKPase in infundibulum. Azo dye method X100

Figure 2 Strong to intense positive reaction AKPase in vagina. Azo dye method X100

Figure 3 Weak to moderate positive reaction G-6-Pase uterovaginal junction. Nitro BT method X100

Figure 4 Weak to moderate positive reaction LDH in uterus. Nitro BT method X100

Figure 5 Weak to moderate positive reaction $\mathrm{SDH}$ in isthmus Nitro BT method X100

Figure 6 Moderate to strong positive reaction G-6-PD in uterus. Nitro BT method X40

Figure 7 Moderate to strong positive reaction NADH in magnum. Nitro BT method X100

Figure 8 Weak to moderate positive reaction NADPH in isthmus. Nitro BT method X100

Figure 9 Moderate to strong positive reaction Estarse in magnum. Napthol acetate method X100

\section{References}

Aire TA, Steinbach J (1976) Histochemical studies of the development of alkaline and acid phosphatase activities in the ovary and oviduct of the fowl (Gallus domesticus). Acta Anatomica $95: 207-217$

Bashir MB, Tanzeela T, Roznza S (2002) Estimation of serum alkaline phosphatase, cholesterol, calcium and phosphorus during pre-laying and laying conditions different strains of chicken. Pakistan Veterinary Journal 22: 94-96

Barka T, Anderson PG (1963) Histochemistry: Theory, Practice and Bibliography. Harper and Row Publisher Inc. New York, USA

Baskt MR, Akuffo V (2007) Alkaline phosphatase activity in the vagina and uterovaginal junction sperm-storage tubules of turkeys in egg production: implication for sperm storage. British Journal of Poultry Science $48: 515-518$

Baskt MR (2011) Role of the oviduct in maintaining sustained fertility in hens Physiology and Endocrinology Symposium. Journal of Animal Science 89: 1323-1329

Fredricssion B (1969) Histochemistry of the oviduct. The mammalian oviduct. (Eds) Hafez E S E and Blandau R J. The University of Chicago Press, Chicago

Khillare GS (2012) Molecular and Biochemical events in sperm storage tubules of oviduct during natural and artificial insemination in Japanese quail. M.V.Sc. thesis submitted to the Indian Veterinary Research Institute,Bareli, India.

Khillare GS, Sastry KVH, Singh RP, Agrawal R, Mohan J (2013) Acid and Alkaline phosphatase enzyme activity in sperm storage tubules in Japanese quail. Indian Journal of Poultry Science 48: $128-130$

Padykula HA (1952) The localization of succinic dehydrogenase in tissue section of the rat. American Journal of Anatomy 91: 107145

Pearse AGE (1972) Histochemistry: Theoretical and Applied. 3rd ed. Churchill, Livingstone, London

Rama Rao AVSS (1994) Textbook of Biochemistry UBS Publishers, New Delhi

Uppal V, Roy KS (2007) Histoenzymatic studies on the oviduct during prenatal life in buffalo (Bubalusbubalis Indian Journal of Animal Science 77: 376-380

Valocky I, Legath J, Lenhardt L, Lazar G, Novotny F (2007) Activity of alkaline phosphatase, acidic phosphatase and nonspecific esterase in the oviduct of puerperal ewes after exposure to polychlorinated biphenyls. Veterinary Medicine 52: 186-192

Yamamoto T, Ozawa H, Nagai H (1985) Histochemical studies of Ca-ATPase, succinate and NAD+ -dependent isocitrate dehydrogenases in the shell gland of laying Japanese quails: with special reference to calcium-transporting cells Histochemistry $83: 221-228$ 